\title{
Diagnostic Accuracy of Visceral Adiposity Index in Patients With Nonalcoholic Fatty Liver Disease: A Systematic Review and Meta-Analysis
}

\author{
Xianhao Yi \\ Third Xiangya Hospital, Central South University \\ Shaihong Zhu ( $\square$ shzhu@csu.edu.cn ) \\ Third Xiangya Hospital, Central South University \\ Liyong Zhu \\ Third Xiangya Hospital, Central South University
}

\section{Research Article}

Keywords: Diagnostic accuracy, Visceral adiposity index, Nonalcoholic fatty liver disease, Meta-analysis

Posted Date: December 13th, 2021

DOI: https://doi.org/10.21203/rs.3.rs-1137007/v1

License: (c) (i) This work is licensed under a Creative Commons Attribution 4.0 International License. Read Full License 


\section{Abstract}

Background: Many studies have shown that visceral adiposity index (VAI) is valuable in the diagnosis of nonalcoholic fatty liver disease (NAFLD), but the accuracy of VAl in the diagnosis of NAFLD is not consistent. we aimed to assess the VAl as a predictor of NAFLD.

Methods: The Cochrane Library, PubMed, Embase, and other databases were searched to collect all the documents that meet the inclusion criteria, The retrieval period is from the establishment of the database to September 2021. The methodological quality of the included studies was evaluated using the quadas- 2 tool. The heterogeneity among the studies was analyzed by the Cochran- $\mathrm{Q}$ test and $\mathrm{I}^{2}$ test, and the appropriate model was selected according to the heterogeneity. The diagnostic efficacy of VAl was evaluated by meta-analysis and a Fagan diagram was drawn to evaluate the diagnostic ability of VAl.

Results: A total of 9 studies were included. The overall quality of the included studies was good. Meta-analysis showed that the combined sensitivity (Sen combined) of VAl in the diagnosis of NAFLD was 0.70 [95\% $\mathrm{Cl}(0.69-0.71)$ ], the combined specificity (Spe combined) was 0.67 [95\% $\mathrm{Cl}$ (0.670.68)], PLR combined was 2.08 [95\% Cl (1.87-2.31)], NLP combined was 0.39 [95\% Cl (0.34-0.44)], and DOR combined was 5.81 [95\% Cl (4.73-7.14)]. The corresponding AUC was 0.79 [95\% $\mathrm{Cl}(0.75-0.82)]$. Meta-regression analysis showed that the gold index was a potential source of heterogeneity (P $<0.05$ ). The Fagan diagram shows that the precision of NAFLD diagnosis is $70 \%$ when the pre-test probability is set to $50 \%$ and then supplemented by VAl.

Conclusion: VAI has a certain value in the diagnosis of NAFLD and may be helpful in the early detection of NAFLD, but it still needed a large number of prospective studies to support this.

\section{Background}

Nonalcoholic fatty liver disease (NAFLD) is an increasingly common disease with a prevalence of $25.24 \%$ worldwide ${ }^{[1]}$. NAFLD is closely related to metabolic syndrome and can cause significant economic and social burdens ${ }^{[2]}$. Early detection of NAFLD is of great importance for its treatment and prognosis, among which liver biopsy is the gold indicator for the diagnosis of NAFLD, but its invasive effect limits its clinical application. Noninvasive diagnostic methods include MRI, CT, NFS, FIB-4, etc., but they have disadvantages such as high cost and complicated indicators ${ }^{[3]}$. Clinical and epidemiological studies have shown that visceral fat is closely related to NAFLD ${ }^{[4]}$. Visceral adiposity index (VAI) is a new scoring system based on waist circumference (WC), body mass index (BMI), triglyceride (TG), and high-density lipoprotein (HDL). VAI is an important index recently proposed to assess the distribution and dysfunction of visceral fat, and has been shown to be closely related to cardiovascular and cerebrovascular risk ${ }^{[5]}$. At present, there is still controversy about whether VAI can accurately distinguish NAFLD. This study systematically evaluated whether VAI can accurately distinguish NAFLD through meta-analysis, aiming to provide a reliable evidence-based medical basis for clinical research.

\section{Methods}

\section{Protocol and registration}

This review was conducted in accordance with the preferred reporting items for systematic reviews and meta-analysis (PRISMA) guidelines ${ }^{[6]}$ and has been registered in the International Prospective Register of Systematic Reviews (PROSPERO; registration number: CRD42021275084).

\section{Search strategy}

Computer retrieval of The Cochrane Library (Issue 2, 2013), PubMed, and Embase. Clinical studies related to THE diagnosis of NAFLD by VAI were searched from database establishment to September 2021. Keywords were combined with free words for retrieval. English search terms include Nonalcoholic Steatohepatitis, nonalcoholic fatty liver disease, metabolic associated fatty liver disease (MAFLD), NASH, NAFLD, MAFLD, Visceral adiposity index, VAl.

\section{Inclusion and exclusion criteria}

Two evaluators independently screened literature, extracted data, and evaluated the methodological quality of the included studies according to the inclusion and exclusion criteria. In case of disagreement, the dispute shall be settled through discussion or adjudicated by a third party. The inclusion criteria were as follows: (1) Published diagnostic studies of VAI in diagnosing NAFLD, And it is a primary or original study; (2) The study population consisted of hospitalized patients who received final imaging or pathological diagnosis. Sample size 30 or more. The true positive value (TP), false positive value (FP), false negative value (FN), and true negative value (TN) were provided or can be calculated statistically ${ }^{[7]}$; (3) The test to be evaluated is VAl, and the imaging results or pathological results are the gold standards; (4) Sensitivity (Sen), specificity (Spe), positive likelihood ratio (PLR), negative likelihood ratio (NLR), diagnostic odds ratio (DOR), and Subject operating Characteristic curve (SROC) and area under the curve (AUC) were calculated. The exclusion criteria were: (1) conference report; (2) Missing important data reports or unclear measurement indicators. (3) Published repeatedly in different journals, When two studies in the same institution reported the same target outcome, the quality of inclusion was better; (4) only those studies whose title was related to VAI diagnosis of NAFLD, but not related to VAI diagnosis of NAFLD. 
Two independent investigators reviewed the full-text articles. The included items were evaluated as yes, no, or uncertain. Inter-researcher disagreements were resolved by consensus, or by a third investigator. Data extraction table was developed to extract data, which mainly included: the purpose of extraction was usually the general information of the study (document number, document title, year, author, statistical method), the information of patients (gender, age, etc.), and the quality evaluation of the literature. Then, the methodological quality of the included studies was evaluated according to the quadAS-2 tool developed by Whiting et al. ${ }^{[8]}$ to evaluate the quality of diagnostic studies.

\section{Statistical analysis}

RevMan 5.3 and Meta-Disc 1.4 software were used for analysis. Spearman correlation analysis was used to investigate whether there was a threshold effect, and the SROC curve was summarized to determine whether the SROC pattern was "shoulder and arm shape". Corran-q test and I2 test were used to analyze the heterogeneity among the included studies. Appropriate models were selected according to the heterogeneity (if $\mathrm{P}>0.05$ and $\mathrm{I} 2<$ $50 \%$, the fixed-effects model was used for meta-analysis; otherwise, a random-effects model was used for meta-analysis). Z-test was used to analyze the area under the SROC curve among subgroups, and the one-by-one exclusion method was used for sensitivity analysis to evaluate the stability of the study results. Stata 16.0 software was used to draw Deek's funnel plot to evaluate publication bias, and Fagan plot was used to evaluate the diagnostic ability of VAI. $\mathrm{P}<0.05$ was considered statistically significant.

\section{Patient and public involvement}

No patients or the public were involved in the research.

\section{Ethics approval statement}

This study did not involve human participants.

\section{Results}

\section{Literature search results}

529 related pieces of literature were initially detected, and 9 studies were finally included after layer by layer screening ${ }^{[9-17]}$. The document screening process and results are shown in Fig. 1.

\section{Basic characteristics and quality evaluation of included studies}

The included studies included 14486 cases and 23702 controls. The basic information of each study is shown in Table 1 . The quadas- 2 evaluation system is used for literature quality evaluation. The results show that the cumulative scores of Li et al. ${ }^{[11]}$ and Musso et al. ${ }^{[9]}$ are 3 points, and the cumulative scores of other studies are $\geq 4$ points, indicating that the overall quality of the included literature is good (Fig. 2 ).

\section{Meta-analysis results}

Spearman rank correlation analysis showed that the sensitivity was positively correlated with (1-specificity) $(r s=0.450, P=0.224)$, and there was no threshold effect. The heterogeneity of VAI was analyzed by Sen, Spe, PLR, NLR, and DOR. The results showed that there was heterogeneity $(P<0.001$,

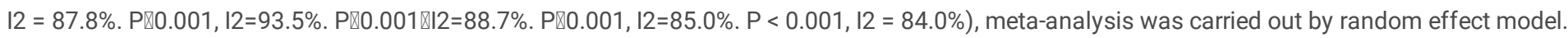
The results showed that the combined sensitivity (Sen combined) of VAl in the diagnosis of NAFLD was 0.70 [95\% $\mathrm{Cl}(0.69-0.71)$ ], the combined specificity (Spe combined) was 0.67 [95\% Cl (0.67-0.68)], PLR combined was 2.08 [95\% Cl (1.87-2.31)], NLP combined was 0.39 [95\% Cl (0.34-0.44)], and DOR combined was 5.81 [95\% $\mathrm{Cl}(4.73-7.14)]$. The corresponding AUC is 0.79 [95\% $\mathrm{Cl}(0.75-0.82)]$, as shown in Figures $3-6[$

\section{Subgroup analysis and meta-regression analysis}

After subgroup analysis based on three factors: gold index, control source, and race, it is found that Sen, Spe, PLR, NLR, and DOR of VAI in the diagnosis of NAFLD with biopsy as the gold index are better than those with imaging examination as the gold standard (Table 2 for details). Metaregression analysis shows that the gold index is a potential source of heterogeneity (Fig. 7 for details).

\section{Sensitivity analysis and publication bias}

The sensitivity analysis was carried out by excluding the included studies one by one. The results showed that the included studies had no significant impact on the results. Deek's funnel plot showed that there was no potential publication bias between included studies $(P=0.17)($ Fig. 8).

\section{Clinical application value}

Draw a Fagan diagram, set the pre-test probability as $50 \%$, and then measure VAl. When VAI $\geq 1.25$, the accuracy of diagnosis of NAFLD is $70 \%$. When VAI $<1.25$, the accuracy of diagnosing NAFLD is $27 \%$ (Fig. 9), suggesting that VAI has good accuracy in diagnosing NAFLD. 


\section{Discussion}

In recent years, with the improvement of living standards, the incidence rate of NAFLD has increased year by year and has been developing towards younger age. It has become one of the hidden threats to human health. Although it is a benign disease, it will develop into cirrhosis and even hepatocellular carcinoma with the development of the disease, and reduce the survival rate of the patients ${ }^{[18]}$. Therefore, early diagnosis of NAFLD is very important for its treatment and prognosis. The study found that VAl is closely related to the incidence of NAFLD and can be used as one of the indicators for screening NAFLD in the general population, but there is no conclusion about its diagnostic efficacy.

Meta-analysis showed that Sen, Spe, PLR, NLP, DOR, and AUC of VAI in the diagnosis of NAFLD were $0.70,0.67,2.08,0.39,5.81$, and 0.79 respectively. It is generally believed that the diagnostic efficacy of AUC is low at 0.50-0.60, medium at 0.70-0.90, higher at $>0.90$, PLR greater than 10.00 , and NLR less than 1.00 , indicating that it can be diagnosed or excluded. From the above analysis, it is found that VAl has medium diagnostic efficiency in diagnosing NAFLD, but it can not accurately diagnose NAFLD.

Heterogeneity is an inevitable problem in meta-analysis. There is heterogeneity caused by the non-threshold effect in this analysis. Through subgroup analysis and meta-regression analysis, it is found that heterogeneity is mainly caused by differences in gold indicators. Sen, Spe, PLR, NLR, and DOR with disease examination as gold indicators are higher than those in the imaging examination subgroup, indicating that the diagnostic efficiency of disease examination is better than that of imaging examination, However, imaging examination is still the main means of clinical diagnosis of NAFLD because of its advantages of non-invasive and high accuracy ${ }^{[19]}$. According to the source of control, the diagnostic odds ratio of a healthy population is higher than that of a non-NAFLD population, indicating that VAI can more accurately diagnose NAFLD when compared with the healthy population. According to race, the diagnostic odds ratio of the European populations is higher than that of the Asian population, indicating that VAl can more accurately diagnose NAFLD in the European populations. VAI is a model based on the clinical data of 315 Italian healthy subjects collected by Marco et al. ${ }^{[5]}$ and established by regression analysis. There are significant differences in body fat distribution between European populations and Asian populations ${ }^{[20]}$. This evidence can explain the differences in the diagnostic efficacy of VAl in different control sources and different races.

In addition, the Fagan diagram shows that when the prior probability is $50 \%$, the posterior probabilities of $\mathrm{VAI} \geq 1.25$ and $\mathrm{VAI}<1.25$ are $70 \%$ and $27 \%$ respectively. That is, it is assumed that the probability of a diagnosis of NAFLD is $50 \%$ according to symptoms and personal tests of doctors. After VAI measurement, if $\geq 1.25$, the accuracy of diagnosis of NAFLD will be increased to $70 \%$. On the contrary, if VAI $<1.25$, the accuracy of diagnosis of NAFLD will be $20 \%$. Therefore, VAI diagnosis of NAFLD has a good clinical application prospect.

\section{Strengths and limitations}

This study has the following advantages: (1) all the included studies adopt strict inclusion criteria, and the overall score is high. (2) Subgroup analysis and meta-regression analysis were used to further evaluate that the analysis results were stable and reliable. (3) Fagan diagram shows that VAI has good clinical value.

However, there are still some deficiencies in this study, which is superior to the limitations of Meta-Analysis: (1) failure to obtain non-braided literature may cause publication bias. (2) Only English documents are included in the included documents, but other Chinese, Japanese, German and other documents are not included, which affects the comprehensiveness of the data. (3) Because the critical value of VAI for NAFLD diagnosis is not unified and affected by different NAFLD diagnostic criteria, it may have a certain impact on the final result.

\section{Conclusion}

In conclusion, VAI has a certain value in the diagnosis of NAFLD and is feasible in clinical application. However, further large samples, multi-center data, and unified NAFLD diagnostic criteria are still needed to verify. With the deepening of follow-up research, VAI will have a wide application prospect.

\section{Abbreviations}

VAl: visceral adiposity index; NAFLD: nonalcoholic fatty liver disease; WC: waist circumference; BMI: body mass index; TG: triglyceride; HDL: highdensity lipoprotein; MAFLD: metabolic associated fatty liver disease; TP: true positive value; FP: false positive value ; FN: false negative value; TN: true negative value; Sen: Sensitivity; Spe: specificity; +LR: positive likelihood ratio; -LR: negative likelihood ratio; DOR: diagnostic odds ratio; SROC: Subject operating Characteristic curve; AUC: area under the curve.

\section{Declarations}

\section{Ethics approval and consent to participate}

Not applicable.

\section{Consent for publication}


Not applicable.

\section{Availability of data and materials}

The datasets used and/or analysed during the current study are available from the corresponding author on reasonable request.

\section{Competing interests}

The authors declare that they have no conflict of interest.

\section{Funding}

This research did not receive any specific grant from funding agencies in the public, commercial, or not-for-profit sectors.

\section{Authors' contributions}

Xianhao Yi: literature search, data extraction, data analysis, manuscript preparation.

Liyong Zhu: study concepts and design, data extraction, critical revision.

Shaihong Zhu: study concepts and design, data extraction, critical revision.

All authors have participated sufficiently in the study and approved the final version.

\section{Acknowledgements}

Not applicable.

\section{References}

1. Younossi Z M, Koenig A B, Abdelatif D, et al. Global epidemiology of nonalcoholic fatty liver disease-Meta-analytic assessment of prevalence, incidence, and outcomes. Hepatology 2016; 64: 73-84.

2. Younossi Z, Anstee Q M, Marietti M, et al. Global burden of NAFLD and NASH: trends, predictions, risk factors and prevention. Nature reviews Gastroenterology \& hepatology 2018; 15: 11-20.

3. Castera L, Friedrich-Rust M, Loomba R. Noninvasive Assessment of Liver Disease in Patients With Nonalcoholic Fatty Liver Disease. Gastroenterology 2019; 156: 1264-81.e4.

4. Visceral fat: a key mediator of steatohepatitis in metabolic liver disease. Hepatology 2008; 48.

5. Amato M C, Giordano C, Galia M, et al. Visceral Adiposity Index: A reliable indicator of visceral fat function associated with cardiometabolic risk. Diabetes Care 2010; 33: 920-2.

6. Mcinnes M D F, Moher D, Thombs B D, et al. Preferred Reporting Items for a Systematic Review and Meta-analysis of Diagnostic Test Accuracy Studies: The PRISMA-DTA Statement. Jama 2018; 319: 388-96.

7. Habibzadeh F, Habibzadeh P, Yadollahie M. On determining the most appropriate test cut-off value: the case of tests with continuous results. Biochemia medica 2016; 26: 297-307.

8. Whiting P F, Rutjes A W, Westwood M E, et al. QUADAS-2: a revised tool for the quality assessment of diagnostic accuracy studies. Ann Intern Med 2011; 155: 529-36.

9. Musso G, Cassader M, Gambino R. Diagnostic accuracy of adipose insulin resistance index and visceral adiposity index for progressive liver histology and cardiovascular risk in nonalcoholic fatty liver disease. Hepatology 2012; 56: 788-9.

10. Fedchuk L, Nascimbeni F, Pais R, et al. Performance and limitations of steatosis biomarkers in patients with nonalcoholic fatty liver disease. Aliment Pharmacol Ther 2014; 40: 1209-22.

11. Li L, You W, Ren W. The ZJU index is a powerful index for identifying NAFLD in the general Chinese population. Acta Diabeto/2017; 54: $905-11$.

12. Vassilatou E, Lafoyianni S, Vassiliadi D A, et al. Visceral adiposity index for the diagnosis of nonalcoholic fatty liver disease in premenopausal women with and without polycystic ovary syndrome. Maturitas 2018; 116: 1-7.

13. Fu C P, Ali H, Rachakonda V P, et al. The ZJU index is a powerful surrogate marker for NAFLD in severely obese North American women. PLoS One 2019; 14: e0224942.

14. Villanueva-Ortega E, Garcés-Hernández M J, Herrera-Rosas A, et al. Gender-specific differences in clinical and metabolic variables associated with NAFLD in a Mexican pediatric population. Ann Hepatol 2019; 18: 693-700.

15. Cen C, Wang W, Yu S, et al. Development and validation of a clinical and laboratory-based nomogram to predict nonalcoholic fatty liver disease. Hepatology international 2020; 14: 808-16. 
16. Lin I T, Lee M Y, Wang C W, et al. Gender Differences in the Relationships among Metabolic Syndrome and Various Obesity-Related Indices with Nonalcoholic Fatty Liver Disease in a Taiwanese Population. Int J Environ Res Public Health 2021; 18.

17. Vural Keskinler M, Mutlu H H, Sirin A, et al. Visceral Adiposity Index As a Practical Tool in Patients with Biopsy-Proven Nonalcoholic Fatty Liver Disease/Nonalcoholic Steatohepatitis. Metab Syndr Relat Disord 2021; 19: 26-31.

18. Younossi Z M. Non-alcoholic fatty liver disease - A global public health perspective. J Hepatol 2019; 70: 531-44.

19. Rinella M E, Sanyal A J. Management of NAFLD: a stage-based approach. Nature reviews Gastroenterology \& hepatology 2016; $13: 196-205$.

20. Liu B, Du Y, Wu Y, et al. Trends in obesity and adiposity measures by race or ethnicity among adults in the United States 2011-18: population based study. Bmj 2021; 372: n365.

\section{Tables}

Table 1 Basic characteristics of included studies

\begin{tabular}{|c|c|c|c|c|c|c|c|c|c|c|c|c|}
\hline Author & Year & country & Gold index & $\begin{array}{l}\text { Control } \\
\text { source }\end{array}$ & Race & $\begin{array}{l}\text { Cases } \\
\text { number }\end{array}$ & $\begin{array}{l}\text { Control } \\
\text { number }\end{array}$ & $\begin{array}{l}\text { Cut- } \\
\text { off }\end{array}$ & TP & FP & $\mathrm{FN}$ & TN \\
\hline Cen $^{1}$ & 2020 & China & Ultrasonographic & $\begin{array}{l}\text { Non- } \\
\text { NAFLD }\end{array}$ & Asian & 6261 & 10207 & 1.59 & 4414 & 3205 & 1847 & 7002 \\
\hline Fedchuk $^{2}$ & 2014 & France & Biopsy & $\begin{array}{l}\text { Non- } \\
\text { NAFLD }\end{array}$ & European & 241 & 83 & 1.25 & 190 & 7 & 51 & 76 \\
\hline $\mathrm{Fu}^{3}$ & 2019 & America & Computed tomography & $\begin{array}{l}\text { Non- } \\
\text { NAFLD }\end{array}$ & European & 40 & 67 & 1.46 & 35 & 42 & 5 & 25 \\
\hline Keskinler $^{4}$ & 2020 & Turkey & Biopsy & Healthy & European & 57 & 57 & 1.78 & 45 & 16 & 12 & 41 \\
\hline $\mathrm{Li}^{5}$ & 2017 & China & Ultrasonographic & $\begin{array}{l}\text { Non- } \\
\text { NAFLD }\end{array}$ & Asian & 7324 & 12480 & 1.89 & 4988 & 4152 & 2336 & 8328 \\
\hline $\operatorname{Lin}^{6}$ & 2021 & China & Ultrasonographic & $\begin{array}{l}\text { Non- } \\
\text { NAFLD }\end{array}$ & Asian & 354 & 410 & - & 293 & 192 & 61 & 218 \\
\hline Musso ${ }^{7}$ & 2012 & Italy & Ultrasonographic & Healthy & European & 41 & 82 & 1.9 & 31 & 23 & 10 & 59 \\
\hline Ortega $^{8}$ & 2019 & Mexico & Ultrasonographic & $\begin{array}{l}\text { Non- } \\
\text { NAFLD }\end{array}$ & European & 36 & 158 & 2.33 & 28 & 75 & 8 & 83 \\
\hline Vassilatou $^{9}$ & 2018 & Greece & Ultrasonographic & $\begin{array}{l}\text { non- } \\
\text { NAFLD }\end{array}$ & European & 132 & 158 & - & 85 & 34 & 47 & 124 \\
\hline
\end{tabular}

Abbreviations: TP, True positive value; FP, False positive value; FN, False negative value; TN, True negative value; NAFLD, Nonalcoholic fatty liver disease.

Table 2 Subgroup and Meta-regression analysis of VAI in the diagnosis of NAFLD in included studies

\begin{tabular}{|c|c|c|c|c|c|c|c|c|}
\hline Subgroup & $\begin{array}{l}\text { Number of } \\
\text { studies }\end{array}$ & Sen $\nabla 95 \% \mathrm{Cl} \otimes$ & Spe $\nabla 95 \% \mathrm{Cl} \rrbracket$ & PLR $₫ 95 \% \mathrm{Cl} \rrbracket$ & NLP $₫ 95 \% \mathrm{Cl} \rrbracket$ & DOR $\triangle 95 \% \mathrm{Cl} \bigotimes$ & $\begin{array}{l}\text { RDOR } \\
\text { 『95\%CI } ~\end{array}$ & $\mathrm{P}$ \\
\hline \multicolumn{9}{|l|}{ gold index } \\
\hline biopsy & $2^{1,2}$ & $\begin{array}{l}0.79 \llbracket 0.74- \\
0.83 \rrbracket\end{array}$ & $\begin{array}{l}0.84 \rrbracket 0.76- \\
0.89 \rrbracket\end{array}$ & $\begin{array}{l}5.00 \rrbracket 1.27- \\
19.72 \rrbracket\end{array}$ & $\begin{array}{l}0.24 \rrbracket 0.19- \\
0.30 \rrbracket\end{array}$ & $\begin{array}{l}19.79 \llbracket 4.8- \\
81.63 \rrbracket\end{array}$ & \multirow[t]{2}{*}{$\begin{array}{l}4.06 \rrbracket 1.87- \\
8.79 \square\end{array}$} & \multirow[t]{2}{*}{0.0036} \\
\hline $\begin{array}{c}\text { imaging } \\
\text { examination }\end{array}$ & $7^{3-9}$ & $\begin{array}{l}0.70 \otimes 0.69- \\
0.70 \rrbracket\end{array}$ & $\begin{array}{l}0.67 \rrbracket 0.67- \\
0.68 \rrbracket\end{array}$ & $\begin{array}{l}1.99 \otimes 1.80- \\
2.19 \rrbracket\end{array}$ & $\begin{array}{l}0.43 \rrbracket 0.39- \\
0.47 \rrbracket\end{array}$ & $\begin{array}{l}4.96 \rrbracket 4.26- \\
5.79 \rrbracket\end{array}$ & & \\
\hline \multicolumn{9}{|l|}{ control source } \\
\hline Healthy & $2^{2,3}$ & $\begin{array}{l}0.78 \rrbracket 0.68- \\
0.85 \square\end{array}$ & $\begin{array}{l}0.72 \varangle 0.64- \\
0.79 \rrbracket\end{array}$ & $\begin{array}{l}2.75 \rrbracket 2.06- \\
3.67 \rrbracket\end{array}$ & $\begin{array}{l}0.31 \varangle 0.21- \\
0.46 \rrbracket\end{array}$ & $\begin{array}{l}8.78 \rrbracket 4.76- \\
16.06 \rrbracket\end{array}$ & \multirow[t]{2}{*}{$\begin{array}{l}1.38 \otimes 0.35- \\
5.40 \rrbracket\end{array}$} & \multirow[t]{2}{*}{0.5898} \\
\hline Non-NAFLD & $7^{1,4-9}$ & $\begin{array}{l}0.70 \otimes 0.69- \\
0.70 \rrbracket\end{array}$ & $\begin{array}{l}0.67 \rrbracket 0.67- \\
0.68 \rrbracket\end{array}$ & $\begin{array}{l}2.01 \rrbracket 1.80- \\
2.25 \rrbracket\end{array}$ & $\begin{array}{l}0.39 ه 0.35- \\
0.45 \rrbracket\end{array}$ & $\begin{array}{l}5.55 \rrbracket 4.48- \\
6.88 \rrbracket\end{array}$ & & \\
\hline \multicolumn{9}{|l|}{ Race } \\
\hline Asian & $3^{4,8,9}$ & $\begin{array}{l}0.70 \otimes 0.69- \\
0.70 \rrbracket\end{array}$ & $\begin{array}{l}0.67 \rrbracket 0.67- \\
0.68 \rrbracket\end{array}$ & $\begin{array}{l}2.04 \rrbracket 1.86- \\
2.24 \rrbracket\end{array}$ & $\begin{array}{l}0.43 \otimes 0.39- \\
0.48 \rrbracket\end{array}$ & $\begin{array}{l}4.84 \rrbracket 4.07- \\
5.75 \rrbracket\end{array}$ & \multirow[t]{2}{*}{$\begin{array}{l}1.70 \otimes 0.69- \\
4.22 \rrbracket\end{array}$} & \multirow[t]{2}{*}{0.2091} \\
\hline European & $6^{1-3,5-7}$ & $\begin{array}{l}0.76 ه 0.72- \\
0.79 \rrbracket\end{array}$ & $\begin{array}{l}0.67 \bigotimes 0.64- \\
0.71 \rrbracket\end{array}$ & $\begin{array}{l}2.66 \otimes 1.61- \\
4.38 \rrbracket\end{array}$ & $\begin{array}{l}0.33 \otimes 0.24- \\
0.45 \rrbracket\end{array}$ & $\begin{array}{l}8.39 \rrbracket 4.38- \\
16.05 \rrbracket\end{array}$ & & \\
\hline
\end{tabular}

Abbreviations: Sen, Sensitivity; Spe, Specificity; PLR, Positive likelihood ratio; NLR, Negative likelihood ratio; DOR, Diagnostic odds ratio; RDOR, Relative diagnostic odds ratio; NAFLD, Nonalcoholic fatty liver disease. 
Figures

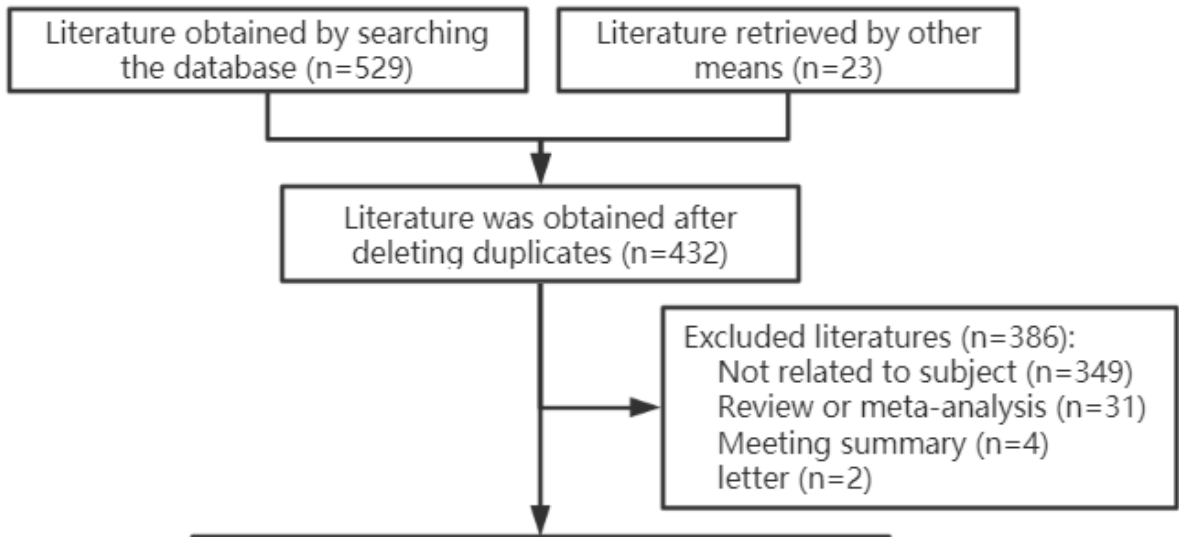

Read the title and abstract and obtain the

literature after preliminary screening $(n=46)$

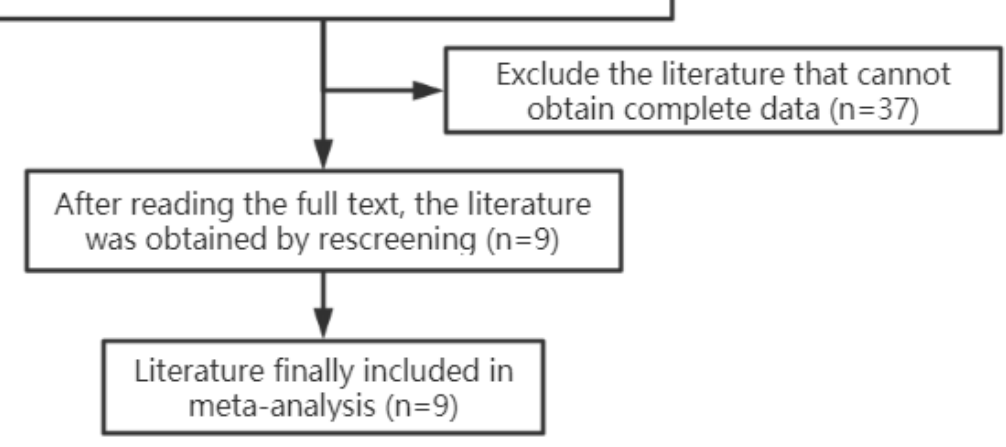

Figure 1

Flow chart of literature screening 


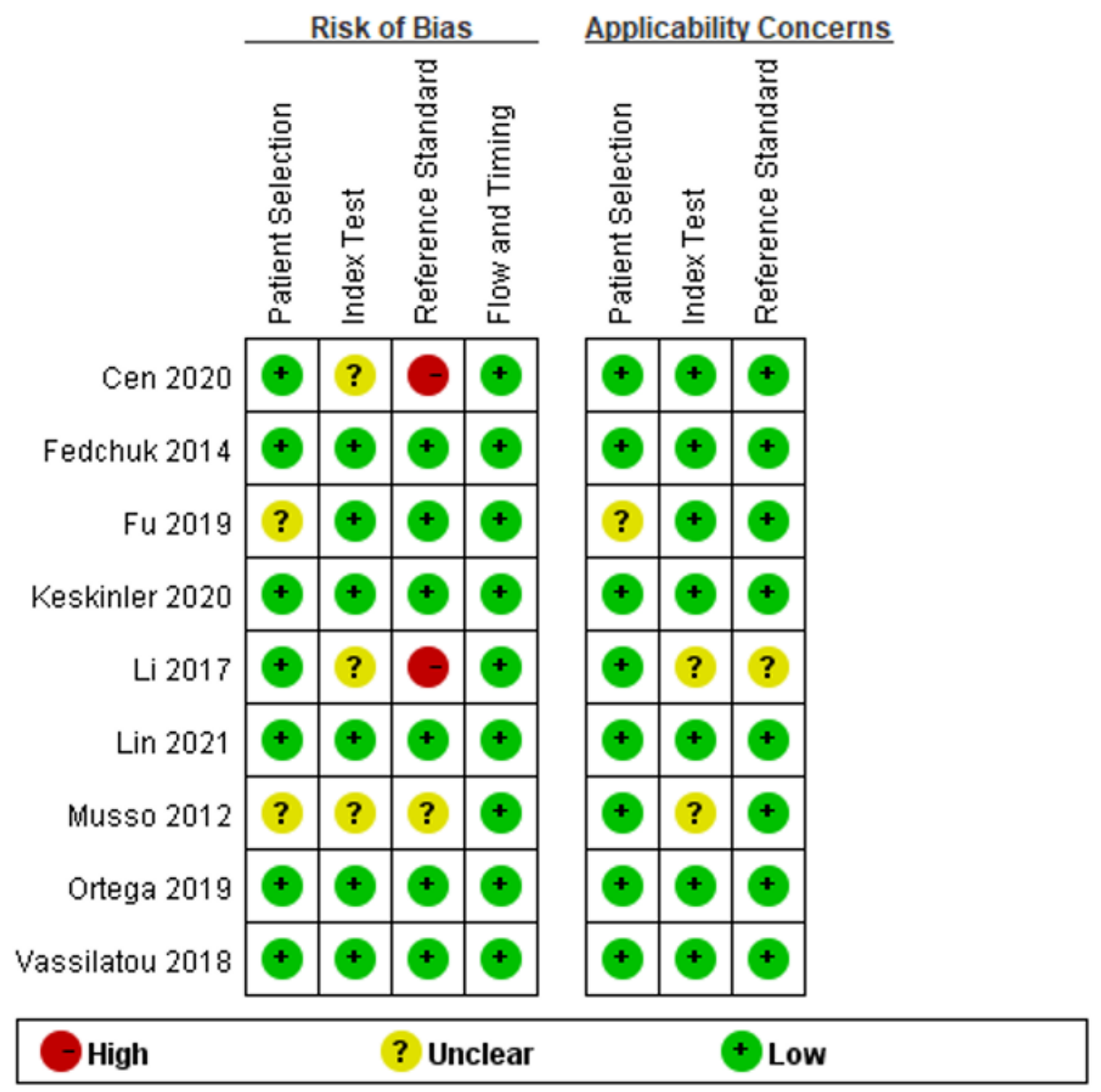

Figure 2

Methodological quality of the included studies assessed by the QUADAS-2 tool

Figure 3

Forest plot assessing the pooled sensitivity and specificity of VAl in the diagnosis of NAFLD in included studies

Figure 4

Forest plot assessing the pooled positive and negative likelihood ratios of VAI in the diagnosis of NAFLD in included studies

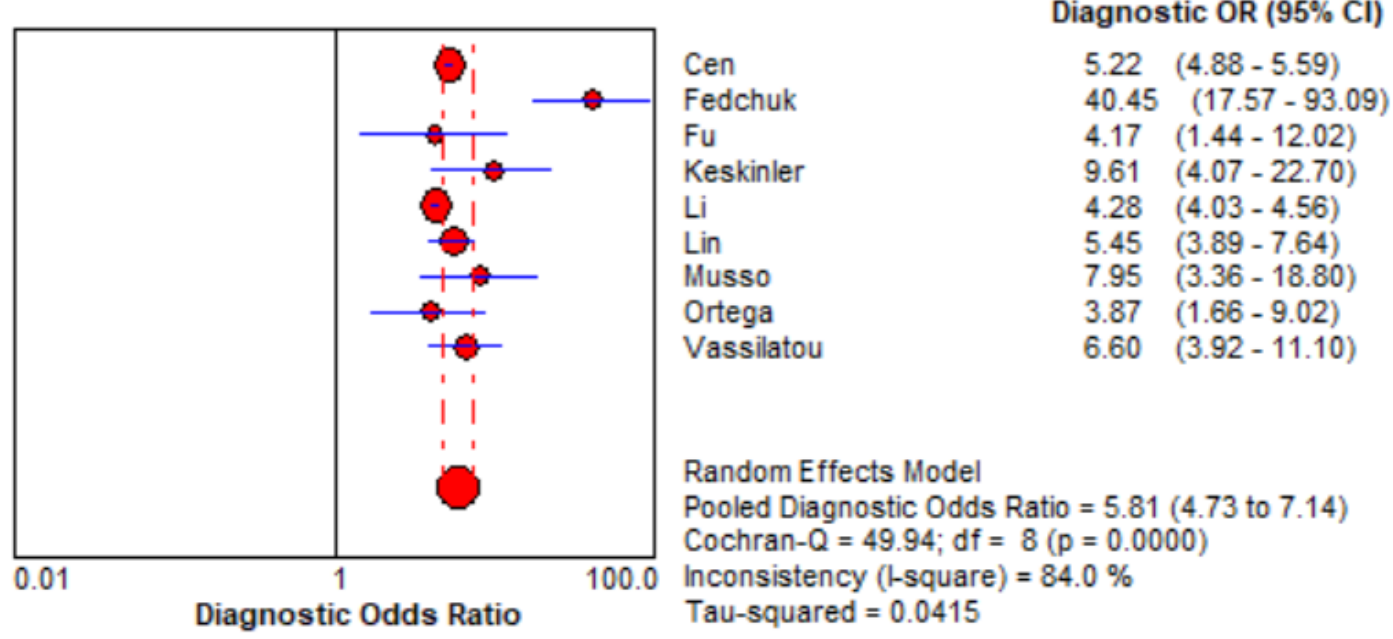




\section{Figure 5}

Forest plot assessing the diagnostic odds ratio of VAl in the diagnosis of NAFLD in included studies

\section{Figure 6}

Summary receiver operating curve of the diagnosis performance of VAI for NAFLD in included studies

\section{Figure 7}

Meta-regression analysis of VAI in the diagnosis of NAFLD in included studies

\section{Figure 8}

Deek's funnel plot assessing the publication bias of included studies

\section{Figure 9}

Fagan's nomogram showing the diagnostic value of VAI for NAFLD 